\title{
Origin of the Atriopeptin-like Immunoreactive Innervation of the Paraventricular Nucleus of the Hypothalamus
}

\author{
David G. Standaert and Clifford B. Saper ${ }^{1}$ \\ Departments of Pharmacology, Neurology, and Anatomy and Neurobiology, Washington University School of Medicine, \\ St. Louis, Missouri 63110, and 'Departments of Pharmacological and Physiological Sciences and Neurology, and the \\ Committee on Neurobiology, University of Chicago, Chicago, Illinois 60637
}

\begin{abstract}
The paraventricular nucleus of the hypothalamus (PVH) contains a prominent collection of varicose atriopeptin-like immunoreactive (APir) fibers. We have used immunohistochemistry and fluorescent retrograde tracers to investigate the origin of these fibers. All parts of the PVH contain APir fibers. The densest collections are found in the periventricular area and in the parvocellular components of the nucleus. Somewhat smaller numbers of fibers are found within the borders of the magnocellular part of the PVH. Following the injection of fluorescent retrograde tracers into the PVH and subsequent immunohistochemical staining, numerous retrogradely labeled APir neurons were observed in the anteroventral periventricular nucleus (AVPV), adjacent to the anteroventral tip of the third ventricle. Smaller groups of retrogradely labeled APir neurons were observed in the ventromedial part of the bed nucleus of the stria terminalis, the pedunculopontine and laterodorsal tegmental nuclei, and the medial part of the nucleus of the solitary tract.

The APir projection from the AVPV to the PVH is of particular interest in view of the abundant evidence implicating both regions in the regulation of the fluid and electrolyte balance and blood pressure. AP may serve as a central neuromodulator as well as a circulating hormone in cardiovascular regulation.
\end{abstract}

Atriopeptin (AP) is a 28 amino acid peptide hormone that is synthesized and secreted by the atria of the heart and is involved in the regulation of fluid and electrolyte balance (Needleman et al., 1985). In addition to its role as a circulating hormone, AP may also be a central neuromodulator (Standaert et al., 1985a, 1987a). Immunohistochemical studies have revealed populations of atriopeptin-like immunoreactive (APir) neurons in the hypothalamus, pontine tegmentum, and medulla (Kawata et al., 1985; Saper et al., 1985; Skofitsch et al., 1985; Standaert et al., 1986a). The presence of this peptide in a number of nuclei that are involved in the central regulation of the cardiovascular system suggests that AP may serve as a central neurotransmitter

\footnotetext{
Received Apr. 22, 1987; revised Oct. 20, 1987; accepted Oct. 20, 1987.

We would like to thank Ms. Quan Hue Ha for technical assistance and Ms. Jane Repovsch fur assistance in preparation of the manuscript. C.B.S. was the recipient of a McKnight Scholar Award. This work was supported by a USPHS Grant NS22835, NIGMS Grants GM07200 and GM07805, a grant in aid from the American Heart Association (850894), the Brain Research Foundation, the Otho Sprague Foundation, and the Washington University-Monsanto Grant.

Correspondence should be addressed to Dr. C. B. Saper, Department of Pharmacological and Physiological Sciences, University of Chicago, 947 East 58th Street, Chicago, IL 60637.

Copyright (C) 1988 Society for Neuroscience $0270-6474 / 88 / 061940-11 \$ 02,00 / 0$
}

as well as a circulating hormone in the regulation of fluid and electrolyte balance (Saper et al., 1985; Standaert et al., 1985a, 1987a).

The paraventricular nucleus of the hypothalamus (PVH) has an important role in the central integration of autonomic control (Swanson and Sawchenko, 1983). The magnocellular part of this nucleus contains the neurosecretory cells responsible for the secretion of vasopressin and oxytocin from the posterior lobe of the pituitary gland. The parvocellular parts of the PVH contain neurons that send descending projections to the nucleus of the solitary tract and to the parasympathetic and sympathetic preganglionic neurons in the medulla and spinal cord (Saper et al., 1976; Swanson and Kuypers, 1980). The medial parvocellular division of the PVH also contains neurons that project to the median eminence (Wiegand and Price, 1980) and synthesize anterior pituitary releasing hormones such as corticotrophinreleasing factor (Swanson and Sawchenko, 1983; Swanson et al., 1983).

The PVH contains a prominent collection of varicose APir fibcrs (Kawata ct al., 1985; Skofitsch et al., 1985; Standaert et al., 1985a,b). In the present study, we have used immunohistochemical methods in combination with retrograde fluorescent tracers to examine the extranuclear origin of APir fibers within the PVH. Additional experiments using a more selective neuronal tracer, wheat germ agglutinin coupled to horseradish peroxidase (WGA-HRP), were performed to examine in more detail the connections between the $\mathrm{PVH}$ and regions containing APir neurons.

\section{Materials and Methods}

Antiserum. The rabbit antiserum to atriopeptin (AP11) used in this study was raised against a 92 amino acid fragment of the high-molecularweight precursor of AP prepared by cyanogen bromide digestion of material purified from rat atria (Geller et al., 1984). Cross-reactivity of this antibody in radioimmunoassay (Schwartz et al., 1985) as well as controls for the specificity of immunohistochemical staining (Saper et al., 1985; Standaert et al., 1986a) have been described previously. Specificity of the antiserum in the present experiments was tested by preadsorbing it with the purified cyanogen bromide fragment of the AP precursor at a concentration of $5 \mu \mathrm{g}$ peptide $/ \mathrm{ml}$ diluted serum. This abolished all neural and cardiac staining.

Fluorescent tracer experiments. Male Sprague-Dawley rats weighing 250-300 gm were anesthetized with chloral hydrate and placed in a stereotaxic apparatus (Kopf). Injections of $25-50 \mathrm{nl}$ of a $5 \%$ solution of either Fast blue or Diamidino yellow dyes (Illing) were made into the region of the paraventricular nucleus using a glass micropipette syringe (Saper, 1983). After survival times of 3-28 d, the animals were reanesthetized, and colchicine ( $150 \mu \mathrm{g}$ in $10 \mu \mathrm{l}$ saline; Sigma) was injected stereotaxically into the lateral ventricle using a Hamilton syringe. Two days later, the animals were deeply anesthetized with chloral hydrate 

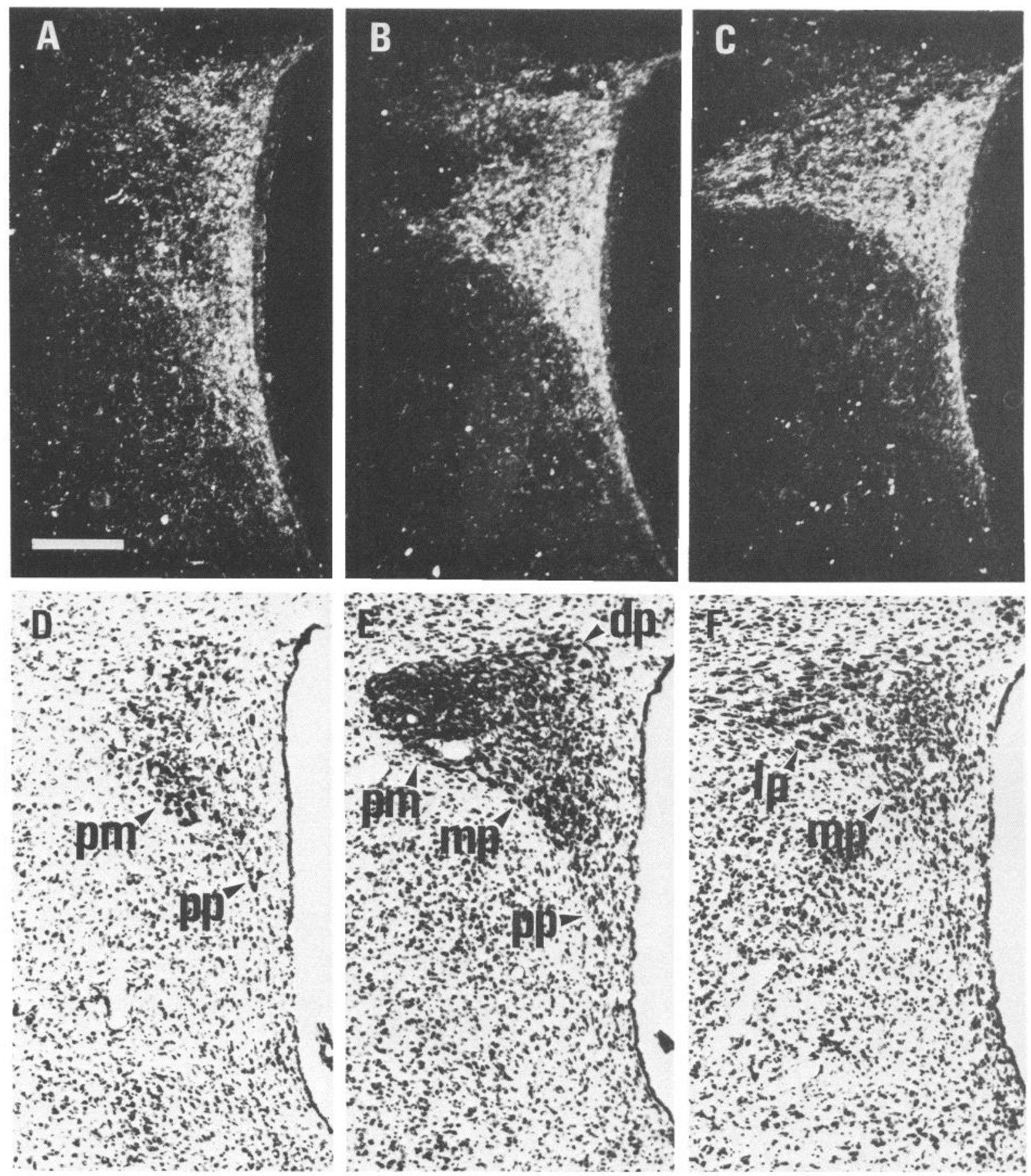

Figure 1. Atriopeptin-like immunoreactive (APir) fibers in the paraventricular nucleus of the hypothalamus (PVH). A-C, Dark-field photomicrographs of a preparation stained with an immunoperoxidase technique demonstrating the organization of APir fibers in PVH at 3 different levels, from rostral $(A)$ to caudal $(C)$. Dense collections of APir fibers are found in the periventricular $(p p)$, medial $(m p)$, dorsal $(d p)$, and lateral $(l p)$ parvocellular parts of the nucleus, while relatively fewer fibers are found within the posterior magnocellular $(p m)$ subnucleus. $D-F$, Thionin-stained sections from another brain, selected to match the rostrocaudal levels of $A-C$. Scale bar, $200 \mu \mathrm{m}$.

and perfused through the heart with $300 \mathrm{ml}$ normal saline, then with $300 \mathrm{ml}$ of $4 \%$ paraformaldehyde in $100 \mathrm{~mm}$ PBS, $\mathrm{pH} 6.5$, and finally with $300 \mathrm{ml}$ of $4 \%$ paraformaldehyde in PBS, pH 8.5. The brain was left in situ for 30-90 $\mathrm{min}$ at room temperature before it was removed and frozen on the stage of a freezing microtome. Fifty micron coronal sections were cut from the level of the anterior tip of the third ventricle to the caudal medulla. In most cases, every third section was stained for APir using the immunofluorescent technique described previously (Standaert et al., 1986a). Ethidum bromide was used as a fluorescent counterstain to reveal neuronal structures (Schmued et al., 1982). Stained sections were mounted on gelatin-coated slides and either coverslipped with glycerin containing $100 \mathrm{~mm}$ Tris, $\mathrm{pH} 8.6$, and $0.2 \%$ paraphenylene diamine or cleared in xylene and coverslipped with DPX containing $2 \% \beta$-mercaptoethanol to retard fluorescent fading.

Sections were studied using a Leitz microscope with Ploem optics and filters $\mathrm{I} 2$ for fluorescein isothiocyanate, N2 for ethidum bromide, and $\mathrm{A}$ for Fast blue and Diamidino yellow. The location of labeled cells was recorded using a Minnesota Datametrics MD1 system coupled to 

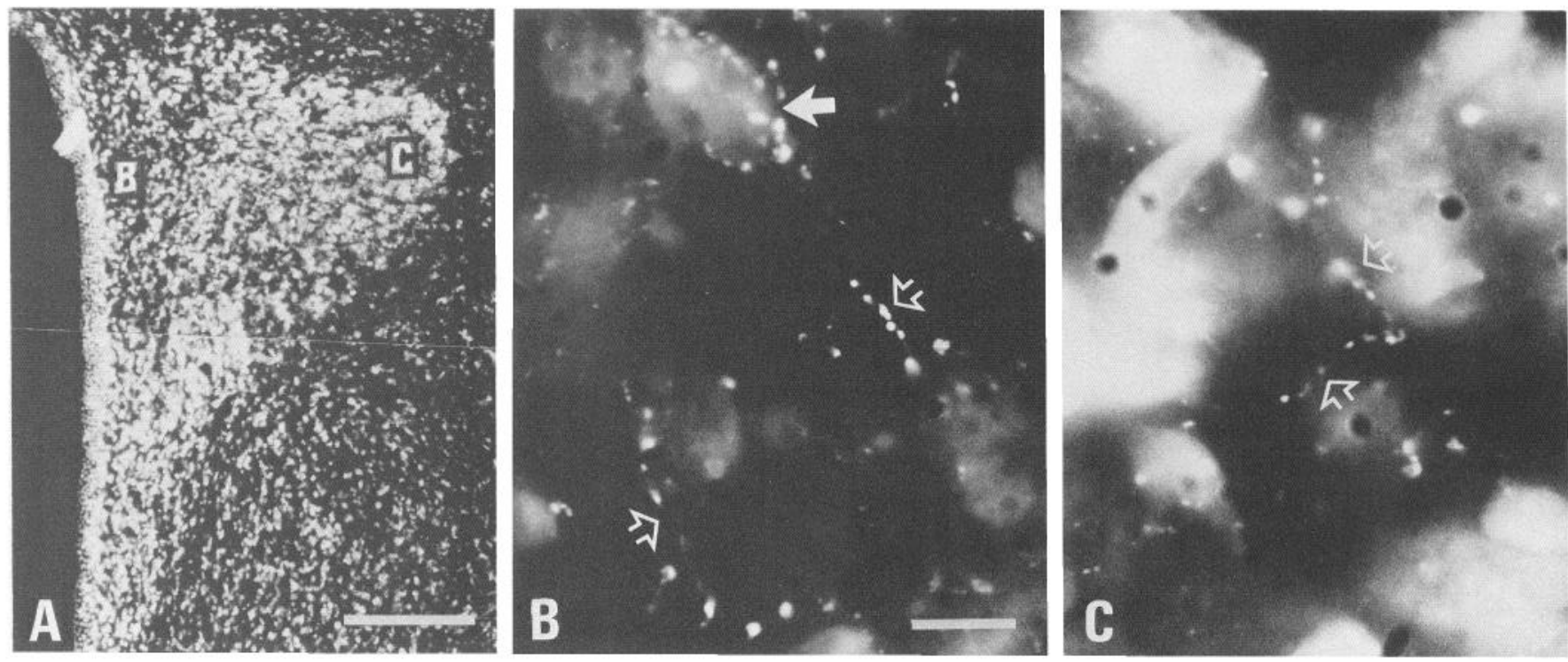

Figure 2. Section through the paraventricular nucleus of a normal rat, stained for APir and counterstained with ethidium bromide. A, Low-power photomicrograph of the red-colored ethidium bromide counterstain, revealed with the Leitz N2 filter system. The areas enclosed by the boxes labeled $B$ and $C$ indicate the regions shown at higher power in the next 2 panels. $B$ and $C$, Small regions of the same section, viewed with the A2 filter system, to reveal the green-colored immunohistochemical staining. $B$ shows numerous punctate varicosities in the periventricular parvocellular part of the PVH (open arrows). Note the dense collection of varicosities surrounding the neuronal cell body in the upper left (solid arrow). C shows similar APir varicosities among the magnocellular neurons in the posterior magnocellular subnucleus of the PVH (open arrows). Scale bars: $A$, 200 $\mu \mathrm{m} ; B$ and $C, 10 \mu \mathrm{m}$.

an $X$ - $Y$ plotter. Sections were then stained with thionin in order to identify tissue landmarks.

WGA-HRP experiments. WGA-HRP ( $1 \%$ in saline, $3-50 \mathrm{nl}$; Sigma) was injected stereotaxically into the region of the PVH in 16 male Sprague-Dawley rats using methods similar to those described above. Two days later, the animals were anesthetized with chloral hydrate and perfused through the heart with saline followed by $0.5 \%$ paraformaldehyde- $1.25 \%$ glutaraldehyde in $\mathrm{PBS}, \mathrm{pH} 7.5$. The brains were removed and stored overnight at $4^{\circ} \mathrm{C}$ in $30 \%$ sucrose. Fifty micron sections were cut on a freezing microtome and processed using the tetramethylbenzidine method of de Olmos et al. (1978). Stained sections were mounted on gelatin-coated slides, counterstained with thionin, and dehydrated rapidly in a graded series of ethanols at $4^{\circ} \mathrm{C}$.

\section{Results}

Distribution of APir fibers in $\mathrm{PVH}$

Specimens from both normal and colchicine-treated animals, prepared in the course of a previous study (Standaert et al., 1986a), as well as sections through the paraventricular region contralateral to the injection site of the animals employed in the present study were examined to determine the distribution of APir fibers within the paraventricular nucleus. The nomenclature employed is consistent with that of Swanson and Kuypers (1980).
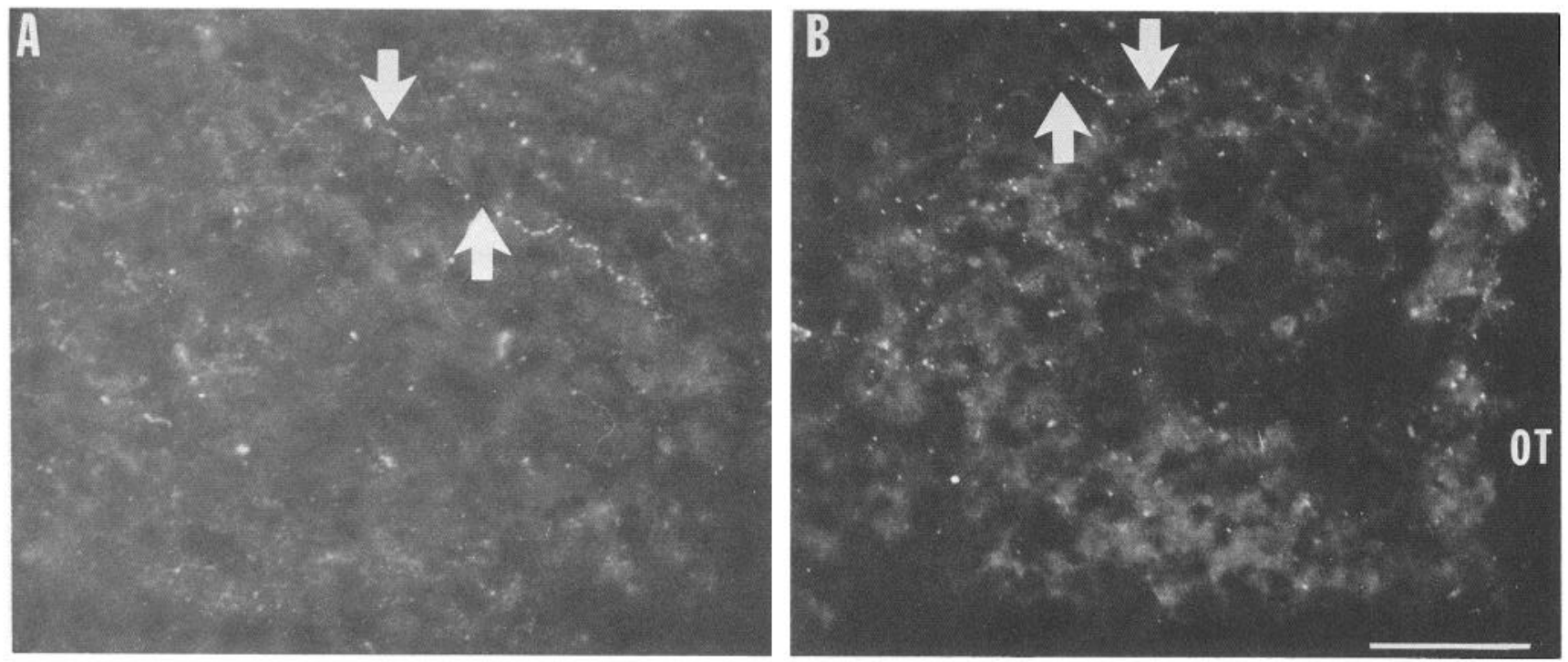

Figure 3. A pair of photomicrographs to illustrate the APir innervation of the posterior magnocellular paraventricular nucleus $(A)$ and the supraoptic nucleus $(B)$. Long immunoreactive fibers with punctate varicosities (arrows) as well as brightly fluorescent boutons are found throughout both of these structures. The density of the APir innervation in these 2 regions is comparable. Scale bar, $50 \mu \mathrm{m}$. 

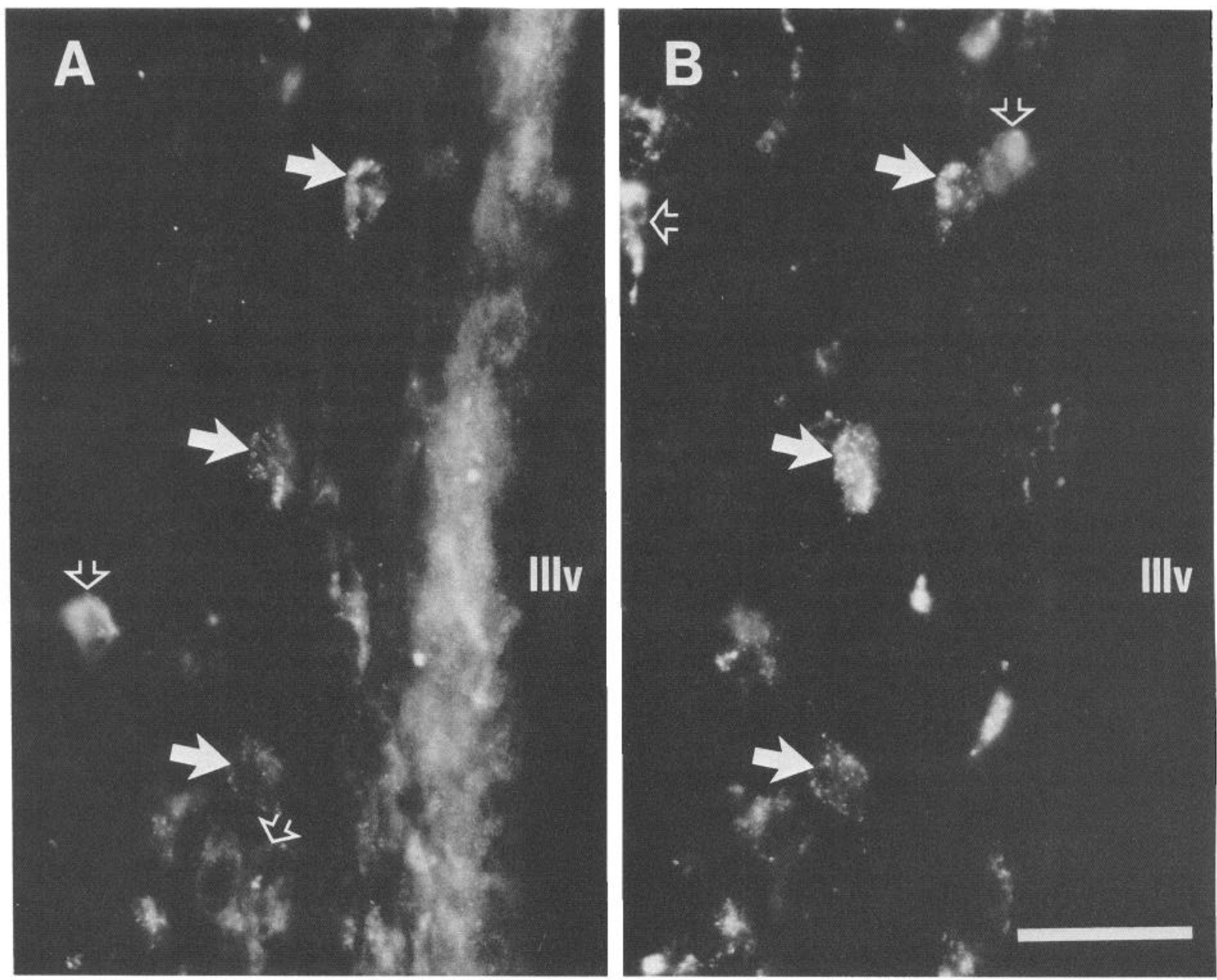

Figure 4. Retrograde labeling of APir neurons in the anteroventral periventricular nucleus (AVPV), following an injection of Fast blue into the PVH. $A$ demonstrates several APir neurons adjacent to the wall of the third ventricle, while $B$ shows the same field viewed with illumination appropriate for the retrograde tracer. By comparing the 2 photomicrographs, it is apparent that at least 3 of the APir neurons in this field also contain the retrograde tracer (solid arrows). Several neurons that are either APir or contain the retrograde tracer, but not both, can also be identified (open arrows). Note also the intense AP immunoreactivity of the ependymal surface $(A)$, typical of this AP antiserum. Scale bar, $50 \mu \mathrm{m}$.

All of the parvocellular components of the PVH contained large numbers of APir fibers. Rostral to the level of the paraventricular system, a network of fine APir fibers was present adjacent to the walls of the third ventricle. Most of these fibers appeared to have a rostrocaudal orientation and had a moderate number of beadlike varicosities. More caudally, the fibers diverged from the wall of the ventricle, forming a dense plexus in the periventricular, medial, and lateral parvocellular subnuclei of the PVH (Fig. 1). The fibers in these parvocellular areas had a tortuous trajectory and were studded with numerous varicosities (Fig. 2). The periventricular region contained a particularly dense collection of fibers, although the density of varicosities was similar to that seen in the medial and lateral parvocellular regions. In addition to these fibers, populations of medium-sized, round APir perikarya were observed in the medial, lateral, and periventricular parvocellular divisions of the PVH, as described previously (Standaert et al., 1986a).

The posterior magnocellular subnucleus of the PVH also contained APir fibers, although the innervation was less intense than that of the parvocellular regions. At low magnification, the posterior magnocellular subnucleus appeared as a relatively sparsely innervated island amidst the dense fiber networks of the parvocellular areas (Fig. 1). At higher magnification, we observed numerous tortuous, varicose fibers within the magnocellular subnucleus (Fig. 2). The density of fibers in the posterior magnocellular region of the PVH was similar to that observed in the supraoptic nucleus, which also contains perikarya of vasopressin- and oxytocin-secreting neurons (Fig. 3, Standaert et al., 1986a). No immunoreactive perikarya were seen in the magnocellular part of the PVH or in the supraoptic nucleus.

In the most caudal part of the PVH, the APir fibers became relatively sparse, consisting mainly of the rostrocaudally oriented fibers along the wall of the ventricle and a small bundle traversing the most caudal part of the lateral parvocellular subnucleus to enter the medial part of the zona incerta. The areas lateral to the PVH throughout its length contained only a few, if any, APir fibers; similarly, relatively few fibers were found dorsal to the PVH. Thus, any substantial extrinsic sources of APir innervation to the $\mathrm{PVH}$ must reach the nucleus by traveling in the periventricular region.

\section{Injections of fluorescent tracers into the PVH}

A total of 12 animals received injections of either Fast blue or Diamidino yellow into a region that included the PVH. All of 

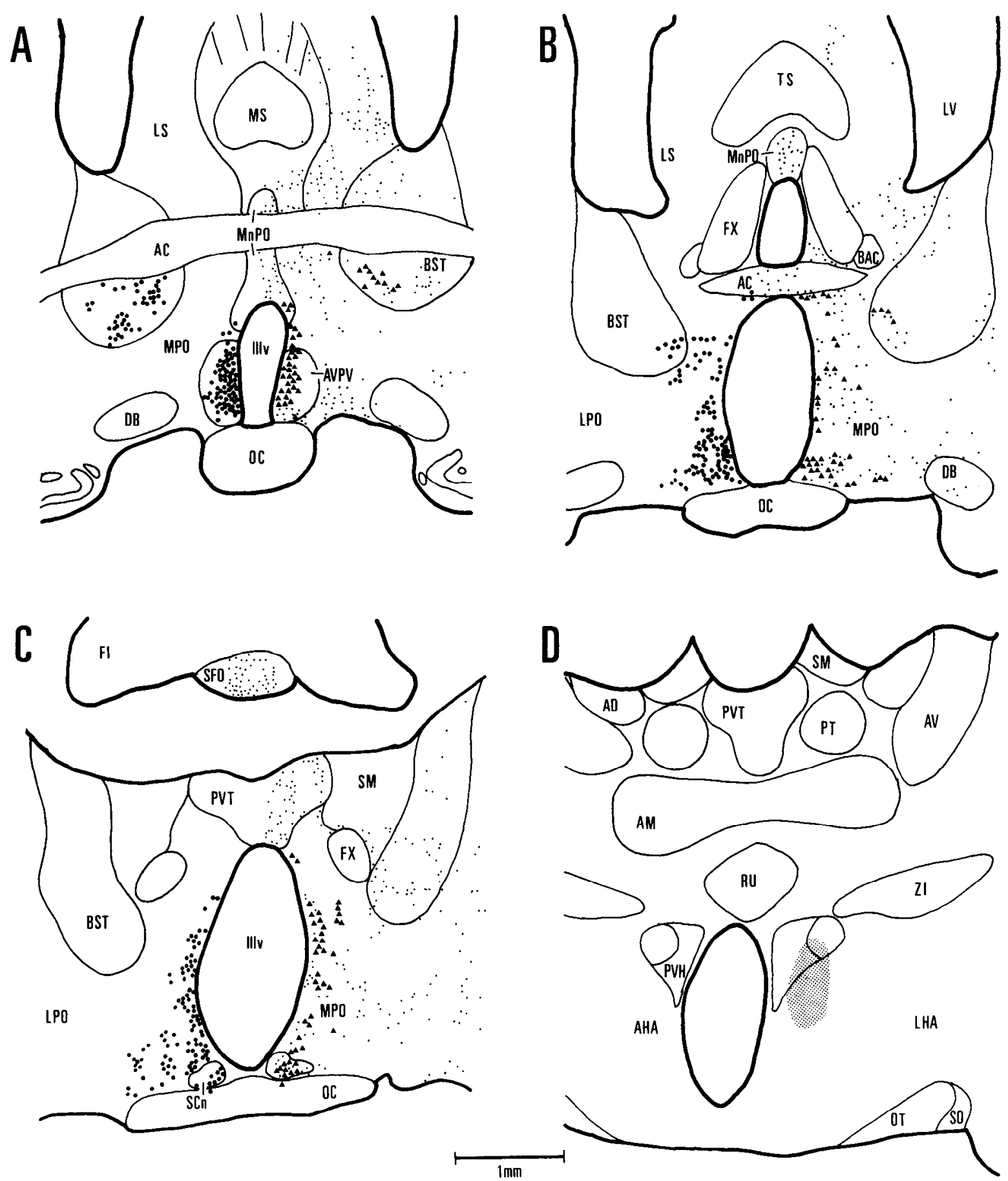

Figure 5. Distribution of APir and retrogradely labeled neurons following an injection of Fast blue into the PVH. In this series of drawings of coronal sections, the large dots on the left indicate the location of all of the APir neurons present at each rostrocaudal level. On the right of each drawing, the triangles indicate the location of APir neurons that were retrogradely labeled by Fast blue, while the small dots indicate the location of Fast blue-labeled neurons that were not APir. $D$ indicates the location of the injection site. See Appendix abbreviations.

these injections included the posterior magnocellular division of PVH, as well as portions of the lateral, medial, and periventricular parvocellular divisions. None of the injections was strictly confined to the PVH, although most included only small portions of the adjacent anterior hypothalamic area, zona incerta, or ventromedial thalamus.

In these animals, several regions were consistently observed to contain retrogradely labeled APir neurons (Fig. 3). The largest collection of these double-labeled neurons, observed in all 12 animals, was associated with the anteroventral tip of the third ventricle. Rostrally, a dense cluster of round or oval APir neurons was found adjacent to the wall of the ventricle just caudal to the organum vasculosum of the lamina terminalis, in the anteroventral periventricular nucleus (AVPV, Fig. 4A; Simerly et al., 1985; Standaert et al., 1986a). Although the density of the APir neurons in this structure is such that it was difficult to 

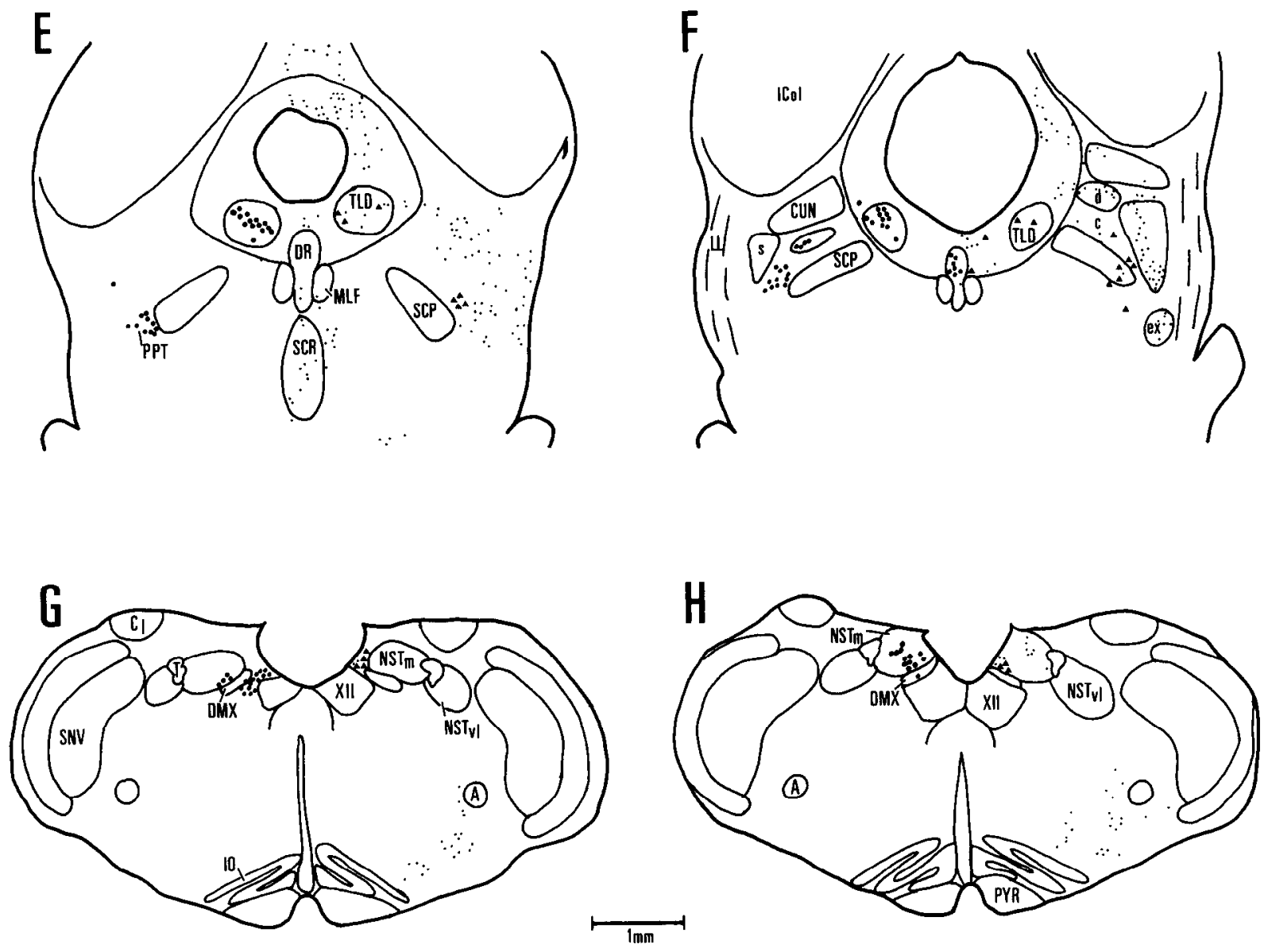

Figure 5. Continued.

count them in $50 \mu \mathrm{m}$ sections, in most cases about one-third to one-half of the APir neurons in the AVPV were retrogradely labeled following injections that included PVH. APir neurons extended caudally from AVPV along the wall of the third ventricle and laterally into the medial preoptic area. A substantial portion of these APir neurons was also retrogradely labeled (Fig. $4, B, C$ ). In most cases, double-labeled neurons were observed in the periventricular area as far caudally as the suprachiasmatic nucleus. Caudal to this level, spread of dye from the injection site during the immunohistochemical staining procedure made it difficult to determine which APir neurons in the periventricular system were retrogradely labeled.

A second, smaller group of APir neurons that were consistently retrogradely labeled was located within the ventromedial part of the bed nucleus of the stria terminalis (BST). Rostrally, this group of double-labeled neurons first appeared just ventromedial to the anterior commissure. The largest numbers of these double-labeled BST neurons were found more caudally, at the level of the crossing of the anterior commissure, where they constituted about $15-20 \%$ of the total number of BST APir neurons (Fig. 4A). Caudal to this level, APir neurons in BST were relatively scarce (Fig. $4, B, C$ ).

Small populations of APir neurons in several additional areas were retrogradely labeled following injections including PVH. Two groups of large, multipolar APir neurons were present in the rostral part of the pons. In an earlier study (Standaert et al., 1986b), we used immunocytochemical techniques to show that these neurons are identical to the cholinergic neurons of the laterodorsal tegmental (TLD; Tohyama et al., 1978) and pedunculopontine (PPT) nuclei (Armstrong et al., 1983; Rye et al., 1987). After each of the injections of the fluorescent tracers into the PVH, about $5-10 \%$ of the APir neurons in both PPT and TLD also contained the retrograde tracer (Fig. $4, E, F$ ). The nucleus of the solitary tract contained a small population of APir neurons, concentrated largely in the rostral end of the medial subnucleus (Fig. 4, $G, H$ ). In each case, a small number of these neurons (typically only 1 or 2 neurons per section) were also retrogradely labeled. Most of these double-labeled neurons were found in the dorsal and rostral parts of the medial subnucleus, adjacent to the floor of the fourth ventricle.

Several additional regions of the brain contained retrogradely labeled neurons after injections into the region of $\mathrm{PVH}$, including the subfornical organ, the lateral septum, and the paraventricular nucleus of the thalamus; however, none of these areas contained APir perikarya. Retrogradely labeled neurons were also observed in the lateral hypothalamus, the mammillary nuclei, and the parabrachial nucleus; although these areas contained populations of APir perikarya, no double labeling was observed.

Three additional animals received injections centered either lateral or ventral to PVH, which did not include the region of PVH. In none of these animals were retrogradely labeled APir neurons observed in the AVPV, the TLD, the PPT, or the nucleus of the solitary tract. One additional animal received an 

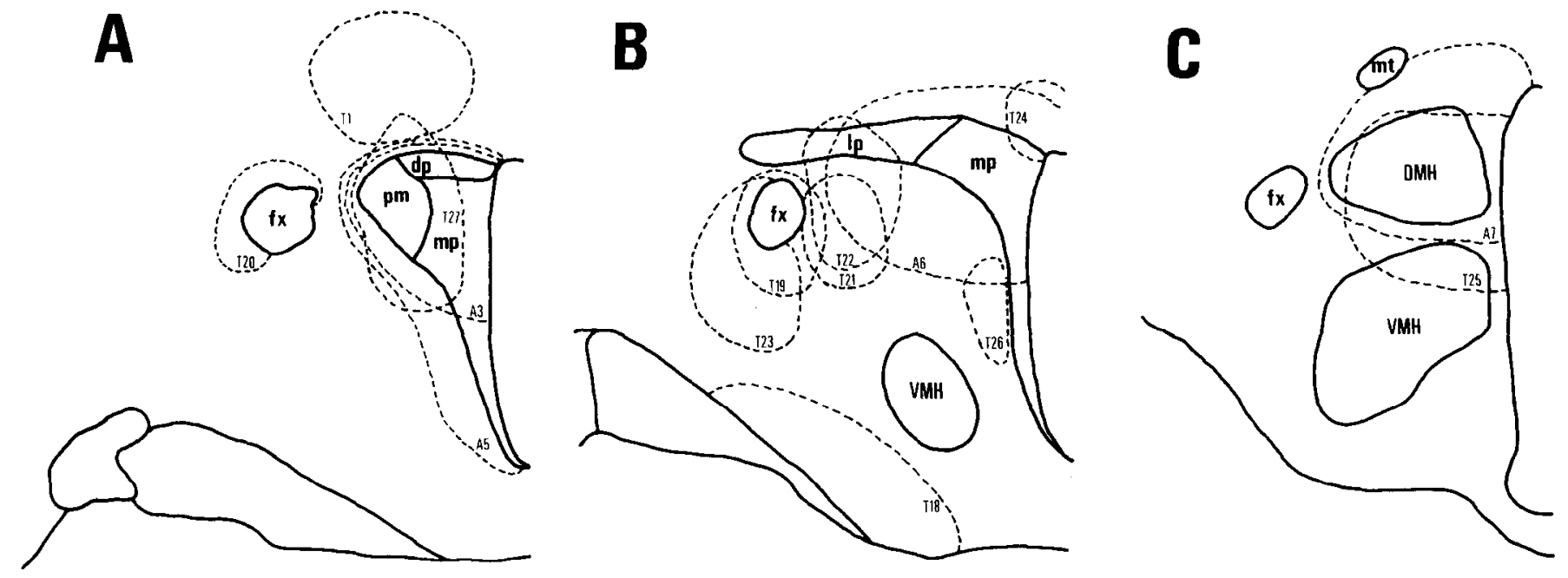

Figure 6. WGA-HRP injection sites in the region of the PVH. In this series of drawings, the dashed lines indicate the area involved by the dense, central core of each injection site, at the level of its maximum extent.

injection of Fast blue into the third ventricle, adjacent to the PVH. Although the ependymal surfaces were brightly labeled, no neuronal labeling was observed in this animal.

\section{Injections of $W G A-H R P$ into the PVH}

The injection sites produced by fluorescent dyes are large, and their borders are not clearly defined. In addition, these dyes may be taken up by fibers passing through the injection site as well as by axon terminals. In order to assess whether the retrograde labeling we had observed with these dyes was due to uptake by terminals within the $\mathrm{PVH}$, we performed a series of experiments using WGA-HRP. This tracer produces small, well-defined injection sites and is not taken up to any great extent by fibers of passage (Brodal et al., 1983; Shook et al., 1984).

Sixteen animals received injections of WGA-HRP in the region of the PVH (Fig. 6). In 6 cases (A3, A5, A6, T22, T27, R163), the central, dense core of the injection site included part or all of the PVH. All 6 of these cases demonstrated retrograde labeling of the previously established afferent projections to the PVH, including the subfornical organ (Miselis, 1981), the median preoptic nucleus (MnPO; Saper and Levisohn, 1983), the parabrachial nucleus (Fulwiler and Saper, 1984), and the medial part of the nucleus of the solitary tract (Ricardo and Koh, 1978; McKellar and Loewy, 1981; Sawchenko and Swanson, 1982).

In each of the animals with injections involving the PVH, large numbers of retrogradely labeled neurons were observed within the borders of the AVPV nucleus, as well as in the ventromedial part of the BST (Fig. 7). In the 10 remaining cases, including injections dorsal, lateral, and ventral to the PVH (Fig. 4), no evidence of retrograde labeling of neurons in the AVPV, the MnPO, or the ventromedial part of the BST was observed. Similarly, cach of the 6 injcctions into PVH produced retrograde labeling of large multipolar neurons in the PPT and the TLD nuclei, at the pontomedullary junction. No labeling was seen in either of these structures following the injections outside of the PVH.

\section{Discussion}

Using an antiserum raised against a fragment of the high-molecular-weight precursor of atriopeptin, we have found that all parts of the PVH are innervated by varicose APir fibers. The largest collections of fibers were found in the parvocellular parts of the PVH, which were innervated by a dense network of tortuous fibers bearing numerous punctate varicosities. The region of the nucleus containing the magnocellular perikarya contained a smaller, but still substantial number of coarse, varicose fibers. Following injections of fluorescent retrograde tracers that included the region of the $\mathrm{PVH}$, we observed a dense cluster of retrogradely labeled APir ncurons in the AVPV nucleus, adjacent to the anteroventral tip of the third ventricle. A second, somewhat smaller group of retrogradely labeled APir perikarya was found in the ventromedial part of the BST, while additional double-labeled neurons were observed in the PPT and TLD nuclei, as well as the medial part of the nucleus of the solitary tract.

The fluorescent dyes we have employed in this study are quite sensitive; thus, these experiments probably delineate the maximum extent of extranuclear APir projections to the PVH. However, there are 2 limitations inherent in the use of these tracers. First, the injection sites are invariably large and cannot be confined entirely to the PVH. Second, these dyes may be picked up by fibers of passage, leading to retrograde labeling of neurons that do not form synapses within the injection site. Therefore, it is important to compare these results to those obtained with other techniques.

Several previous studies have employed HRP as a retrograde tracer to examine the afferent connections of the PVH (Berk and Finkelstein, 1981; Silverman et al., 1981; Tribollet and Dreifuss, 1981). Following injections of HRP into PVH, all 3 groups of investigators observed collections of retrogradely labeled neurons in the preoptic region, although it is not clear from the published descriptions whether these neurons were within AVPV. Silverman et al. (1981) observed retrogradely labeled neurons in the ventromedial part of the BST, while both Berk and Finkelstein (1981) and Tribollet and Dreifuss (1981) found retrogradely labeled neurons in the region of the PPT and TLD nuclei and the medial part of the nucleus of the solitary tract. In the present report, we have described a series of experiments employing WGA-HRP, a tracer that produces small injection sites and is not taken up by undamaged fibers of pas- 

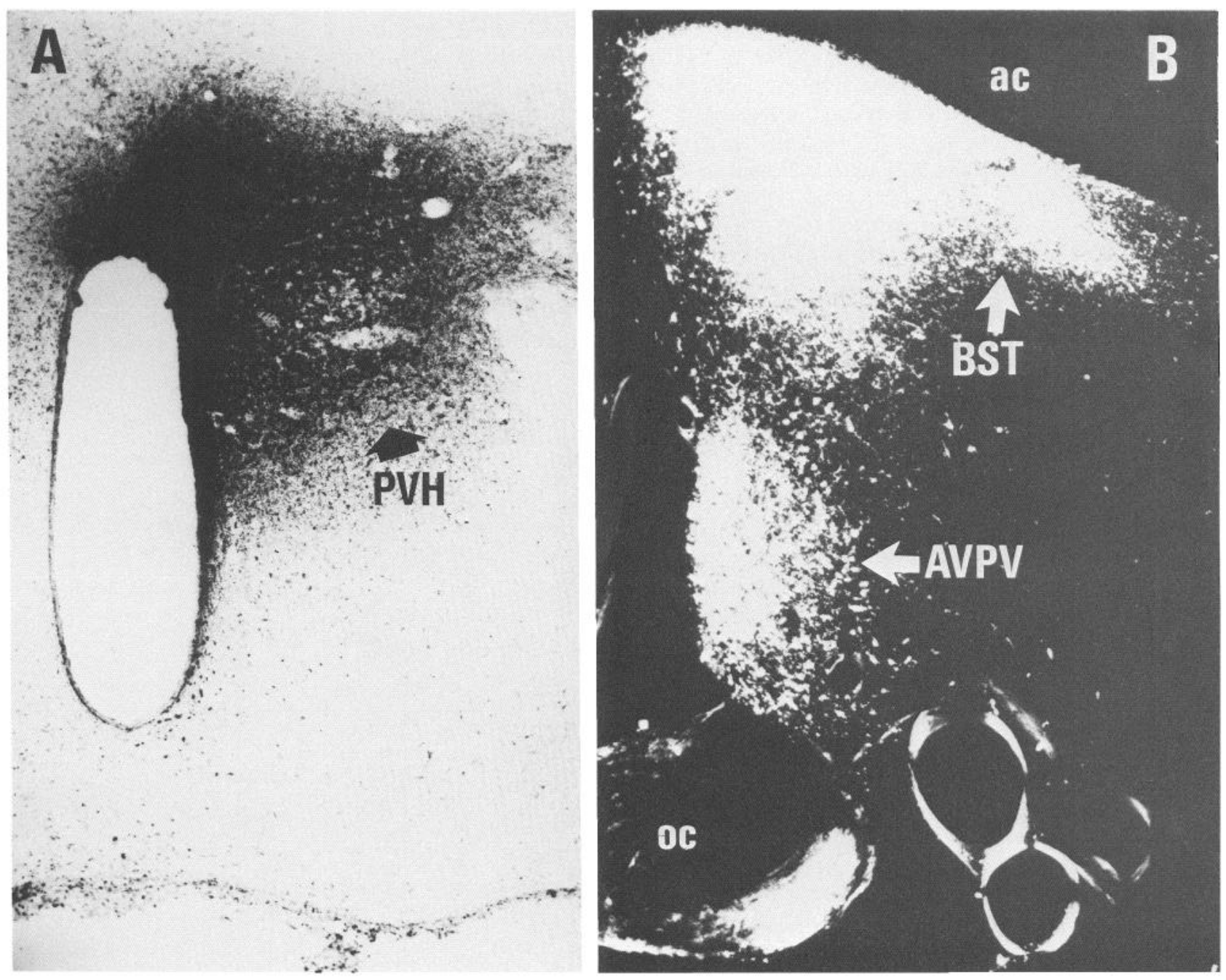

Figure 7. Retrograde labeling of neurons in the preoptic area, following an injection of WGA-HRP into the PVH (R163, not included in Fig. 4). $A$, Bright-field photomicrograph demonstrating the injection site in the PVH. $B$, Dark-field photomicrograph showing labeled neurons within AVPV, as well as the ventromedial part of the bed nucleus of the stria terminalis (BST).

sage (Brodal et al., 1983; Shook et al., 1984). We observed retrograde labeling of neurons in AVPV following injections of WGA-HRP into the PVH, while no labeling was observed following injections into adjacent areas. We have also confirmed the previously described projections to PVH from the BST, the PPT, the TLD, and the nucleus of the solitary tract.

Some of the afferent connections to the PVH have been identified using anterograde tracing techniques. Injections of tritiated amino acids that include the AVPV nucleus and adjacent periventricular area result in the autoradiographic labeling of a terminal field in the PVH, concentrated largely within the periventricular parvocellular part of the nucleus (Conrad and Pfaff, 1976; Swanson, 1976; Sawchenko and Swanson, 1983; Brown et al., 1985). Following small injections of Phaseolus vulgaris lectin (PHA-L) confined entirely to the AVPV nucleus, we have traced individual fibers caudally from AVPV along the wall of the third ventricle into the paraventricular nucleus, where they turn laterally and ramify, forming varicose terminations within the magnocellular and medial, lateral, and periventricular parvocellular parts of the PVH (Brown et al., 1985). Injections of tritiated amino acids that include the ventral part of the BST also produce autoradiographic labeling of the $\mathrm{PVH}$, with most of the silver grains concentrated over the parvocellular parts of the nucleus, while smaller numbers of grains are found in the magnocellular part of the PVH (Swanson and Cowan, 1979; Sawchenko and Swanson, 1983). Autoradiographic studies of efferents from the nucleus of the solitary tract (Ricardo and Koh, 1978; McKellar and Loewy, 1981; Sawchenko and Swanson, 1982) demonstrate a prominent projection to $\mathrm{PVH}$, which involves primarily the parvocellular regions but includes a sparse projection to the magnocellular subnucleus.

Little is known about the projections from the PPT and TLD to the hypothalamus. In our own autoradiographic studies, we observed only a sparse projection from the PPT and the TLD to the PVH. Much larger numbers of labeled fibers were observed dorsal and lateral to the PVH in the zona incerta (Saper and Loewy, 1982; and C. B. Saper, unpublished observations). Similar results have been obtained in recent studies using PHA-L (A. Hallanger, unpublished observations). Following injections into the PPT or the TLD, a small number of labeled varicose fibers were observed within the PVH, while a much larger population of labeled fibers was found lateral and dorsal to the borders of the PVH.

In summary, the results of both anterograde and retrograde 
tracing experiments support the existence of direct projections to PVH from AVPV, the ventromedial part of the BST, the PPT and TLD nuclei, and the medial part of the nucleus of the solitary tract. As each of these areas contained retrogradely labeled APir neurons after injections of fluorescent tracers into PVH, each may contribute to the APir terminal field in that nucleus. In addition to these extranuclear projections, some of the APir fibers in the PVH may be intranuclear in origin. APir perikarya are present in the medial, lateral, and periventricular parvocellular parts of PVH (Kawata et al., 1985; Saper et al., 1985; Skofitsch et al., 1985; Standaert et al., 1986a). Studies employing the Golgi method have established the existence of intranuclear connections within the PVH (Van den Pol, 1982). In the present study, most of the APir perikarya in the parvocellular parts of the PVH ipsilateral to the injection site were labeled by direct diffusion of the tracer, precluding an analysis of intranuclear projections.

An interesting feature of the distribution of APir fibers within the PVH is that there are fewer APir fibers within the borders of the magnocellular subnucleus than there are in the parvocellular regions. The functional significance of this difference is uncertain. It should be emphasized that substantial numbers of APir fibers and terminals are present within the borders of the posterior magnocellular subnucleus. Although they are fewer in number than those in the parvocellular parts of $\mathrm{PVH}$, the density is comparable to that found in the supraoptic nucleus, a structure that, like the magnocellular subnucleus, contains the perikarya of vasopressin- and oxytocin-secreting neurons (Standaert et al., 1986a). Furthermore, the dendrites of the vasopressin and oxytocin neurons in the PVH extend well beyond the borders of the posterior magnocellular subnucleus (Sofroniew and Glasman, 1981; Hou-Ya et al., 1986) into the densely innervated parvocellular regions. Thus, the function of both magnocellular and parvocellular PVH neurons may be affected by direct synaptic input from APir fibers. The possibility of polysynaptic effects mediated by intranuclear connections (Van den Pol, 1982) must also be considered.

Several recent studies have provided evidence supporting the hypothesis that AP within the CNS participates in the regulation of fluid and electrolyte balance. Dietary salt and dehydration can produce alterations in the amount of immunoassayable AP in discrete brain regions (Tanaka et al., 1984; Samson, 1985a). Intracerebroventricular administration of AP in conscious animals can suppress basal as well as dehydration, hemorrhage, or angiotensin-stimulated release of vasopressin (Samson, 1985b; Iitake et al., 1986; Yamada et al., 1986; Samson et al., 1987) and can suppress the drinking response to angiotensin II (Antunes-Rodrigues et al., 1985; Nakamura et al., 1985; Katsuura et al., 1986; Masotto and Negro-Villar, 1986). Iontophoretic or pressure application of AP appears to inhibit the spontaneous electrical activity of some neurons in the region of the paraventricular nucleus (Haskins et al., 1986; Wong et al., 1986). Recently, we have observed that microapplication of femtomole amounts of AP onto physiologically identified paraventricular vasopressin neurons can inhibit the activity of these neurons (Standaert et al., 1987b).

The existence of an APir projection to the PVH from the AVPV is of particular interest because this nucleus lies within the anteroventral third ventricular, or "AV3V" region, an area thought to be important in the neural regulation of blood pressure and fluid and electrolyte balance (see Brody and Johnson, 1980, for review). Animals with lesions in this region show a number of behavioral and hormonal deficits, including alter- ations in drinking behavior, resistance to both renin-dependent and non-renin-dependent models of hypertension (Brody and Johnson, 1980; Johnson, 1985), and abnormal vasopressin responses to osmotic, but not volume, stimuli (Brody and Johnson, 1980; Gardiner et al., 1985; Johnson, 1985). Our data indicate that AP may serve as a neuromodulator linking the AV $3 \mathrm{~V}$ area with the endocrine and autonomic control mechanisms of the PVH (Swanson and Sawchenko, 1983).

\section{Appendix}

\section{List of abbreviations}

$A \quad$ nucleus ambiguus

$A C \quad$ anterior commissure

$A D \quad$ anterodorsal nucleus of the thalamus

$A H A \quad$ anterior hypothalamic area

$A M$

APir

$A V$

$A V P V$

$B A C$

$B S T$

Cl

$C U N$

$d$

$D B$

$D M H$

$D M X$

$d p$

$D R$

ex

FI

$F X$

ICol

IO

LHA

$l p$

$L P O$

$L S$

$L V$

$M L F$

$m p$

$M P O$

$M n P O$

$M S$

$m t$

NSTm

NSTVl

$O C$

$O T$

$P B$

$\mathrm{pm}$

$p p$

$P T T$

$P V H$

PVT

$P T$

PYT

$R U$

$s$

$S C n$

$S C P$

$S C R$

$S F O$

$S M$

$S N V$

SO

$T$

$T L D$

TS

$V M H$

ZI

$I I I v$

$X I I$ anteromedial nucleus of the hypothalamus

atriopeptin-like immunoreactivity

anteroventral nucleus of the thalamus

anteroventral periventricular nucleus

bed nucleus of the anterior commissurc

bed nucleus of the stria terminalis

lateral cuneate nucleus

cuneiform nucleus

$\mathrm{PB}$, dorsal lateral subnucleus

nucleus of the diagonal band

dorsomedial nucleus of the hypothalamus

dorsal motor nucleus of the vagus

PVH, dorsal parvocellular subnucleus

dorsal raphe nucleus

$\mathrm{PB}$, external lateral subnucleus

fimbria

fornix

inferior colliculus

infcrior olivary complex

lateral hypothalamic area

PVH, lateral parvocellular subnucleus

lateral preoptic area

lateral septal nucleus

lateral ventricle

$\mathrm{PVH}$, medial parvocellular subnucleus

medial preoptic area

median preoptic nucleus

medial septal nucleus

mammillothalamic tract

nucleus of the solitary tract, medial subnucleus

nucleus of the solitary tract, ventrolateral subnucleus

optic chiasm

optic tract

parabrachial nucleus

$\mathrm{PVH}$, posterior magnocellular subnucleus

PVH, periventricular parvocellular subnucleus

pedunculopontine tegmental nucleus

paraventricular nucleus of the hypothalamus

periventricular nucleus of the thalamus

paratenial nucleus

pyramidal tract

nucleus reuniens

PB, superior lateral subnucleus

suprachiasmatic nucleus

superior cerebellar peduncle

superior central raphe nucleus

subfornical organ

stria medularis

spinal trigeminal nucleus

supraoptic nucleus

solitary tract

laterodorsal tegmental nucleus

triangular nucleus of the septum

ventromedial nucleus of the hypothalamus

zona incerta

third ventricle

hypoglossal nucleus medial longitudinal fasciculus 


\section{References}

Antunes-Rodrigues, J., S. M. McCann, L. C. Rogers, and W. K. Samson (1985) Atrial natriuretic factor inhibits dehydration and angiotensin II-induced water intake in the conscious, unrestrained rat. Proc. Natl. Acad. Sci. 82: 8720-8723.

Armstrong, D. M., C. B. Saper, A. I. Levey, B. H. Wainer, and R. D. Terry (1983) Distribution of cholinergic neurons in rat brain: Demonstrated by the immunocytochemical localization of choline acetyltransferase. J. Comp. Neurol. 216: 53-58.

Berk, M. L., and J. A. Finkelstein (1981) Afferent projections to the preoptic area and hypothalamic regions in the rat. Neuroscience 6 : 1601-1624.

Brodal, P. E., E. Dietrichs, J. G. Bjaale, T. Nordby, and F. Walberg (1983) Is lectin-coupled horseradish peroxidase taken up and transported by undamaged as well as by damaged fibers in the central nervous system? Brain Res. 278: 1-9.

Brody, M. J., and A. K. Johnson (1980) Role of the $\Lambda$ V3V region in fluid and electrolyte balance, arterial pressure regulation, and hypertension. In Frontiers in Neuroendocrinology, Vol. 6, L. Martini and W. F. Ganong, eds., pp. 249-292, Oxford U. P., London.

Brown, E. R., D. G. Standaert, and C. B. Saper (1985) Efferent connections of the medial preoptic region in the rat. Soc. Neurosci. Abstr. 11: 827 .

Conrad, L. C. A., and D. W. Pfaff (1976) Efferents from medial basal forebrain and hypothalamus in the rat. I. An autoradiographic study of the medial preoptic area. J. Comp. Neurol. 169: 185-220.

de Olmos, J., H. Hardy, and L. Heimer (1978) The afferent connections of the main and accessory olfactory bulb connections in the rat: An experimental HRP study. J. Comp. Neurol. 181: 213-244.

Fulwiler, C. E., and C. B. Saper (1985) Subnuclear organization of the efferent connections of the parabrachial nucleus in the rat. Brain Res. Rev. 7: 229-259.

Gardiner, T. W., J. G. Verbalis, and E. M. Stricker (1985) Impaired secretion of vasopressin and oxylocin in rats after lesions of the nucleus medianus. Am. J. Physiol. 249: R681-R688.

Geller, D. M., M. G. Currie, N. R. Seigel, K. F. Fok, S. P. Adams, and P. Needleman (1984) The sequence of an atriopeptigen: A precursor of the bioactive atrial peptides. Biochem. Biophys. Res. Commun. 121:802-807.

Haskins, J. T., G. J. Zingaro, and R. W. Lappe (1986) Rat atriopeptin III alters hypothalamic neuronal activity. Neurosci. Lett. 67: 279284.

Hou-Ya, A., A. T. Lamme, E. A. Zimmerman, and A. J. Silverman (1986) Comparative distribution of vasopressin and oxytocin neurons in the rat brain using a double label procedure. Neuroendocrinology 44: 235-246.

Iitake, K., L. Share, J. T. Crofton, D. P. Brooks, Y. Ouchi, and E. H. Blaine (1986) Central atrial natriuretic factor reduces vasopressin secretion in the rat. Endocrinology 119: 438-440.

Johnson, A. K. (1985) Role of the periventricular tissue surrounding the anteroventral third ventricle (AV3V) in the regulation of body fluid homeostasis. In Vasopressin, R. W. Schrier, ed., pp. 319-331, Raven, New York.

Katsuura, G., M. Nakamura, K. Inouye, M. Kono, K. Nakao, and H. Imura (1986) Regulatory role of atrial natriuretic polypeptide in water drinking in rats. Eur. J. Pharmacol. 121: 285-287.

Kawata, M., K. Nakao, N. Morri, Y. Kiso, H. Yamashita, H. Imura, and Y. Sano (1985) Atrial natriuretic polypeptide: Topographical distribution in the rat brain hy radioimmunoassay and immunohistochemistry. Neuroscience 16: 521-546.

Masotto, C., and A. Negro-Villar (1985) Inhibition of spontaneous or angiotensin II-stimulated water intake by atrial natriuretic factor. Brain Res. Bull. 15: 523-526.

McKellar, S., and A. D. Loewy (1981) Organization of some brain stem afferents to the paraventricular nucleus of the hypothalamus in the rat. Brain Res. 217: 351-357.

Miselis, R. (1981) The efferent connections of the subfornical organ of the rat: A circumventricular organ within a neural network subserving water balance. Brain Res. 230: 1-23.

Nakamura, M., G. Katsuura, K. Nakao, and H. Imura (1985) Antidipsogenic action of $\alpha$-human atrial natriuretic polypeptide administered intracerebroventricularly in rats. Neurosci. Lett. 58: 1-6.

Needleman, P., S. P. Adams, B. R. Cole, M. G. Currie, D. M. Geller M. L. Michener, C. B. Saper, D. Schwartz, and D. G. Standaert (1985) Atriopeptins as cardiac hormones. Hypertension 7: 469-482.

Ricardo, J. A., and E. T. Koh (1978) Anatomical evidence of direct projections from the nucleus of the solitary tract to the hypothalamus, amygdala, and other forebrain structures in the rat. Brain Res. 153: $1-26$.

Rye, D. B., C. B. Saper, H. J. Lee, and B. H. Wainer (1987) The pedunculopontine nucleus of the rat: Cytoarchitecture, cytochemistry, and some extrapyramidal connections of the mesopontine tegmentum. J. Comp. Neurol. 259: 483-528.

Samson, W. K. (1985a) Dehydration-induced alterations in rat brain vasopressin and atrial natriuretic factor activity. Endocrinology 117 : $1279-1281$.

Samson, W. K. (1985b) Atrial natriuretic factor inhibits dehydration and hemorrhage induced vasopressin release. Neuroendocrinology 40: $277-279$

Samson, W. K., M. C. Aguila, J. Matinovic, J. Antunes-Rodrigues, and M. Norris (1987) Hypothalamic action of atrial natriuretic factor to inhibit vasopressin release. Peptides $8: 449-454$.

Saper, C. B. (1983) A simple inexpensive and reliable nanoliter syringe. Brain Res. Bull. 10: 403-405.

Saper, C. B., and D. Levisohn (1983) Afferent connections of the median preoptic nucleus in the rat: Anatomical evidence for a cardiovascular integrative mechanism in the anteroventral third ventricular (AV3V) region. Brain Res. 288: 21-31.

Saper, C. B., and A. D. Loewy (1982) Projections of the pedunclulopontine tegmental nucleus in the rat: Evidence for additional extrapyramidal circuitry. Brain Res. 252: 367-372.

Saper, C. B., A. D. Loewy, L. W. Swanson, and W. M. Cowan (1976) Direct hypothalamoautonomic connections. Brain Res. 117: 305312.

Saper, C. B., D. G. Standaert, M. G. Currie, D. M. Geller, and P. Needleman (1985) Atriopeptin immunoreactive neurons in the brain: Presence in cardiovascular regulatory areas. Science 227: 1047-1049.

Sawchenko, P. E., and L. W. Swanson (1982) The organization of noradrenergic pathways from the brainstem to the paraventricular and supraoptic nuclei in the rat. Brain Res. Rev. 4: 275-325.

Sawchenko, P. E., and L. W. Swanson (1983) The organization of forebrain afferents to the paraventricular and supraoptic nuclei of the rat. J. Comp. Neurol. 218: 121-144.

Schmued, C., L. W. Swanson, and P. E. Sawchenko (1982) Some fluorescent counterstains for neuroanatomical studies. J. Histochem. Cytochem. 30: 123-128.

Schwartz, D., D. M. Geller, P. T. Manning, N. R. Seigel, K. F. Fok, C. E. Smith, and P. Needleman (1985) Ser-Leu-Arg-Arg-Atriopeptin III: The major circulating form of atrial peptide. Science 229:397400 .

Shook, B. L., B. P. Abramson, and L. M. Chalupa (1984) An analysis of the transport of WGA-HRP in the cat's visual system. J. Ncurosci. Methods 11: 65-77.

Silverman, A. J., D. L. Hoffman, and E. A. Zimmerman (1981) The descending afferent connections of the paraventricular nucleus of the hypothalamus. Brain Res. Bull. 6: 47-61.

Simerly, R. B., L. W. Swanson, and R. A. Gorsky (1985) The distribution of monoaminergic cells and fibers in a periventricular preoptic nucleus involved in the control of gonadotropin release: Immunohistochemical evidence for a dopaminergic sexual dimorphism. Brain Res. 330: 55-64.

Skofitsch, G., D. M. Jacobowitz, R. I. Eskay, and N. Zamir (1985) Distribution of atrial natriuretic factor-like immunoreactive neurons in the rat brain. Neuroscience 16:917-948.

Sofroniew, M. V., and W. Glasman (1981) Golgi-like immunoperoxidase staining of hypothalamic magnocellular neurons that contain vasopressin, oxytocin, or neurophysin in the rat. Neuroscience 6:619643.

Standacrt, D. G., C. B. Saper, and P. Needleman (1985a) Atriopeptin: Potent hormone and potential neuromediator. Trends Neurosci. 8: 509-511.

Standaert, D. G., E. R. Brown, P. Needleman, and C. B. Saper (1985b) Origin of the atriopeptin-immunoreactive innervation of the paraventricular nucleus of the hypothalamus. Soc. Neurosci. Abstr. 11: 827.

Standaert, D. G., P. Needleman, and C. B. Saper (1986a) Organization of atriopeptin-like immunoreactive neurons in the central nervous system of the rat. J. Comp. Neurol. 253: 315-341.

Standaert, D. G., C. B. Saper, D. Rye, and B. H. Wainer (1986b) Colocalization of atriopeptin- with choline acetyltransferase- and substance P-like immunoreactivity in the pedunculopontine and laterodorsal tegmental nuclei in the rat. Brain Res. 382: 163-168.

Standacrt, D. G., P. Nccdlcman, and C. B. Saper (1987a) Atriopeptin: 
Neuromediator in the central regulation of cardiovascular function. In Frontiers in Neuroendocrinology, Vol. 10, L. Martini and W. F. Ganong, eds. (in press)

Standaert, D. G., D. F. Cechetto, P. Needleman, and C. B. Saper (1987h) Atriopeptin inhibits the activity of physiologically identified vasopressin neurons. Nature 329: 151-153.

Swanson, L. W. (1976) An autoradiographic study of the efferent connections of the preoptic region in the rat. J. Comp. Neurol. 167:227256.

Swanson, L. W., and W. M. Cowan (1979) The connections of the septal region in the rat. J. Comp. Neurol. 186: 621-656.

Swanson, L. W., and H. G. J. M. Kuypers (1980) The paraventricular nucleus of the hypothalamus: Cytoarchitectonic subdivisions and organization of projections to the pituitary, dorsal vagal complex, and spinal cord as demonstrated by retrograde fluorescent double-label methods. J. Comp. Neurol. 194: 555-570.

Swanson, L. W., and P. E. Sawchenko (1983) Hypothalamic integration: Organization of the paraventricular and supraoptic nuclei. Ann. Rev. Neurosci. 6: 269-324.

Swanson, L. W., P. E. Sawchenko, J. Rivier, and W. W. Vale (1983) Organization of ovine CRF immunoreactive cells and fibers in the rat brain: An immunohistochemical study. Neuroendocrinology 36 : $165-186$.
Tanaka, I., K. S. Misono, and T. Inagami (1984) Atrial natriuretic factor in rat hypothalamus, atria, and plasma: Determination by specific immunoassay. Biochem. Biophys. Res. Commun. 124: 663-668.

Tohyama, M., K. Satoh, T. Sakumoto, Y. Kimoto, Y. Takahshi, and K. Yamamoto (1978) Organization and projections of neurones in dorsal tegmental area of the rat. J. Hirnforsch. 19: 165-178.

Tribollet, E., and J. J. Dreifuss (1981) Localization of neurones projecting to the hypothalamic paraventricular nucleus area of the rat: A horseradish peroxidase study. Neuroscience 6: 1315-1328.

Van den Pol, A. N. (1982) The magnocellular and parvocellular paraventricular nucleus of the rat: Intrinsic organization. J. Comp. Neurol. 206: 317-345.

Wiegand, S. J., and J. L. Price (1980) Cells of origin of the afferent fibers to the median eminence in the rat. J. Comp. Neurol. 192: 119.

Wong, M., W. K. Samson, C. A. Dudley, and R. L. Moss (1986) Direct, neuronal action of atrial natriuretic factor in the rat brain. Neuroendocrinology 44: 49-53.

Yamada, T., K. Nakao, N. Morri, H. Itoh, S. Shiono, S. Sakamoto, A. Sugawara, Y. Saito, H. Ohno, A. Kanai, G. Katsuura, M. Eigyo, A. Matsushita, and H. Imura (1986) Central effect of atrial natriuretic polypeptide on angiotensin II-stimulated vasopressin secretion in conscious rats. Eur. J. Pharmacol. 125: 453-456. 